\title{
Cycle du développement et PMA : les pays les plus pauvres bénéficieront-ils de la libéralisation des échanges?
}

\author{
Françoise GÉRARD \\ CIRAD, Unité de recherche Normes et régulation \\ des marchés agricoles (Nomade), \\ Fax : 0143947311 \\ <francoise.gerard@cirad.fr>
}

\begin{abstract}
The current trade negotiations' round has been called the development round in order to emphasize ambitious objectives in terms of poverty alleviation: halving the number of poor by 2015. However estimates from world economic models show little improvement for the Less Developed Countries. Moreover several models assumptions may overestimate gains and underestimate losses, especially in the poorest countries where numerous markets imperfections hold. It seems then necessary to avoid that poverty reduction be restricted to emerging countries that development policies and their funding in $L D C$ be discussed simultaneously to trade negotiations
\end{abstract}

Key words: development round, poverty, markets imperfections, world economic models, trade negotiations
Le cycle de négociation en cours à l'OMC a été déclaré Cycle du Développement et la déclaration ministérielle de Doha (novembre 2001) expose sans ambiguïté l'objectif que les négociations commerciales bénéficient aux pays en développement et tout particulièrement aux pays les moins avancés (PMA), dont les difficultés particulières dans l'insertion dans le commerce mondial sont reconnues ${ }^{1}$. II doit permettre, du fait des milliards de dollars de gains obtenus par le retrait des distorsions au commerce international, d'atteindre les objectifs du millénaire et en particulier de diviser par deux le nombre de pauvres.

L'argumentation $^{2}$ est la suivante : les pauvres sont concentrés dans les zones rurales, la plupart vivent des activités agricoles, généralement sous un statut d'indépendant. Le développement agricole apparaît donc comme un levier essentiel pour le développement et la réduction de la pauvreté. Dans ce contexte, les politiques de soutien agricoles des pays de I'OCDE, en déprimant les prix mondiaux, sont particulièrement dommageables car elles se traduisent par des revenus plus faibles pour les agriculteurs pauvres. Ce raisonnement semble solide. Il est par ailleurs étayé par de nombreuses évaluations chiffrées, qui visent à évaluer le niveau des gains et à identifier les gagnants et

\footnotetext{
${ }^{1}$ Première page de la déclaration ministérielle, WTO [1].

2 Développée par exemple par Hertel et Winter [2], p. 4.
}

les perdants. Cependant, non seulement les résultats des modèles mondiaux laissent espérer peu de bénéfices pour les pays les plus pauvres et peu de progrès en termes de réduction de la pauvreté, mais les hypothèses réalisées dans ces modèles sont de nature à surestimer ces gains. En effet, de nombreuses caractéristiques des pays les moins avancés, comme la faiblesse des infrastructures et l'importance des coûts de transaction qui en découle, ainsi que la forte instabilité des prix sur les marchés de produits agricoles, sont de nature à s'opposer aux gains d'efficacité attendus suite à la libéralisation des échanges. Ces éléments ne sont généralement pas inclus dans les modèles. Enfin, ceux-ci ignorent par construction les coûts des ajustements. II semble alors nécessaire, si l'on veut progresser dans la direction indiquée par les Objectifs du Millénaire, et éviter que les pays à faibles revenus soient de plus en plus marginalisés, de ne pas $s^{\prime}$ en tenir aux seules politiques commerciales et d'utiliser la boîte « développement » pour mettre en place de réelles politiques aux bénéfices des populations des pays à faible revenu.

Après avoir rappelé dans une première partie les résultats des modèles mondiaux en matière de réduction de la pauvreté, on s'interrogera sur les raisons d'une telle faiblesse des gains. Les principales caractéristiques des modèles mondiaux et leurs impacts sur les résultats (tendance à la surestimation les gains et à la minimisation des pertes) seront l'objet de la dernière partie.

\section{Niveau et répartition des gains engendrés par le cycle de Doha : des résultats peu favorables aux pays les plus pauvres}

Parallèlement aux négociations sur la libéralisation des échanges, de nombreuses évaluations chiffrées des gains et de leur répartition, à la fois entre les nations et à l'intérieur de celles-ci, ont été réalisées. Les faits stylisés suivants peuvent être déduits de ces études :

- Les milliards de dollars de gains agrégés associés à la libéralisation totale des échanges iront principalement aux pays développés. L'essentiel de ces gains provient du secteur agricole ${ }^{3}$, ils représentent bien peu en termes de croissance des revenus. En effet, si ceux-ci étaient uniformément distribués au sein des pays en développement, et dans l'estimation relativement optimiste du modèle Linkage de la Banque mondiale, les gains atteindraient moins de $20 \$$ par an. Cela fait bien peu au regard de l'étendue du problème de la pauvreté (tableau 1).

- L'essentiel des gains pour les pays en développement ira aux pays émergents (Chine, Inde, Brésil, Argentine).

\footnotetext{
${ }^{3}$ Ceci s'explique par le fait que les taxes sur le commerce extérieur sont beaucoup plus importantes pour les produits agricoles, le commerce des biens manufacturés ayant été plus ou moins libéralisé par le passé.
} 


\section{Encadré 1}

\section{Les gains de la libéralisation sont solubles dans l'incertitude}

Le « modèle international dynamique pour l'étude du développement durable et de la distribution des revenus » (ID ${ }^{3}$ ) a été développé par une équipe de chercheurs du Cirad et de l'Inra [22] avec pour ambition d'évaluer d'un point de vue dynamique l'impact de la libéralisation en prenant en compte, contrairement à la plupart des modèles couramment utilisés, l'instabilité endogène des marchés agricoles, l'imperfection de l'information et ses conséquences sur le comportement des agents.

Construit sur 13 régions, 17 secteurs (dont 14 liés à la production ou la transformation des produits agricoles au sens large) et 5 facteurs de production, il s'agit d'un modèle récursif qui s'inscrit dans la philosophie des modèles d'équilibre général calculable.

Les simulations réalisées, dont un exemple est donné en figure 1, ont permis de mettre en évidence la très forte sensibilité des résultats à l'hypothèse d'imperfection des marchés.

Dans le graphique de gauche, les hypothèses standard de fonctionnement parfait des marchés ont été adoptées, au contraire, dans le graphique de droite le même scénario de libéralisation des échanges est testé dans un modèle où les imperfections des marchés agricoles sont prises en compte. Les gains massifs obtenus à gauche se transforment alors en une succession de pertes et de gains.

Pour l'Afrique subsaharienne, l'impact de la volatilité des marchés réduit à néant les gains de la libéralisation et induit une très forte variabilité du PIB. Dans l'ensemble, les ménages pauvres sont les plus touchés que les ménages riches dans les périodes de dépression et bénéficient moins de la croissance dans les épisodes favorables.

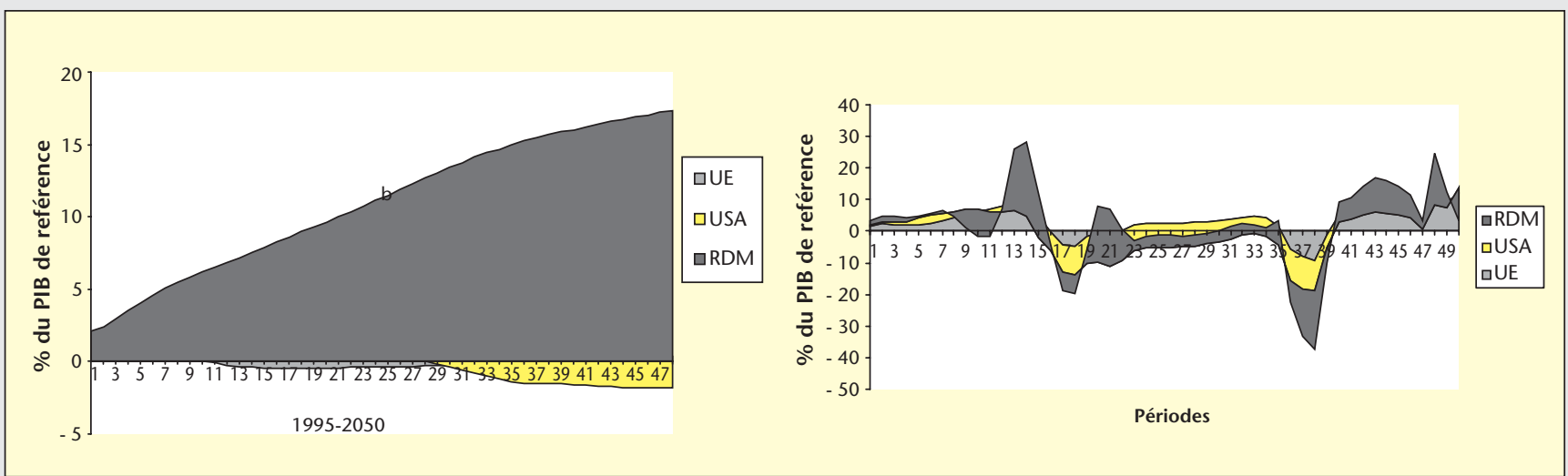

Figure 1. Ces graphiques sont issus de Boussard et al. [23]. Des résultats détaillés peuvent être consultés dans Ayouz et al. [24].

- Les pays les plus pauvres d'Afrique subsaharienne sont susceptibles d'être perdants, du fait des impacts négatifs de la croissance des prix agricoles mondiaux sur les urbains (en particulier, les urbains pauvres) pour des nations qui ne sont pas autosuffisantes.

Les tableaux ci-joints illustrent ce propos en ce qui concerne deux des plus populaires de ces modèles : Linkage, élaboré par la Banque mondiale, et GTAP, élaboré par un groupe d'universitaires de l'université de Purdue, sous la direction de Thomas Hertel.

L'écart entre les résultats de ces deux modèles s'explique par des hypothèses différentes sur les élasticités et la mobilité de la terre entre secteurs agricoles (Anderson et al. 2006, p. 387-95). Par ailleurs, le modèle Linkage représente l'évolution de l'économie mondiale en dynamique récursive alors que le modèle GTAP-Agr est statique. Les divergences importantes dans les résultats obtenus soulignent cependant la difficulté de ce type d'évaluation et la prudence avec laquelle il faut considérer les résultats chiffrés de la libéralisation des échanges. Ce point sera détaillé dans la dernière partie de cet article.
Alors que, considéré globalement les pays en développement bénéficient d'environ $20 \%$ des gains mondiaux d'après le modèle GTAPAgr, les PMA représentés subissent des pertes nettes (tableau 2). Ceci s'explique simplement par leur position d'importateur net de produits

agricoles. L'analyse détaillée des impacts en fonction des mesures de libéralisation confirme cette intuition : le groupe de pays « autre Afrique subsaharienne » perd principalement du fait de la suppression des subventions à l'exportation. Au niveau de l'accès au marché, les

Tableau 1. Niveau et répartition entre groupes de nations des gains de la libéralisation totale des échanges.

\begin{tabular}{|lccccccc|}
\hline Source & Base & $\begin{array}{c}\text { Gains } \\
\text { PED }\end{array}$ & $\begin{array}{c}\text { Gains } \\
\text { pays riches }\end{array}$ & $\begin{array}{c}\text { Gains } \\
\text { monde }\end{array}$ & Gains PED & $\begin{array}{c}\text { Gains } \\
\text { pays riches }\end{array}$ & $\begin{array}{c}\text { Gain total } \\
\text { monde }\end{array}$ \\
\hline \multicolumn{3}{c}{ Milliards de \$ } & \multicolumn{3}{c}{ \$ par tête } \\
\hline GTAP & 2001 & 22 & 62 & 84 & 4,26 & 64,58 & 13,70 \\
Linkage & 2001 & 85,7 & 201,6 & 287,3 & 17,41 & 205,21 & 46,82 \\
\hline
\end{tabular}

Sources : GTAP AGR : Hertel et Keeney [3] ; Linkage : Anderson et al. [4].

Tableau 2. Répartition des gains de la libéralisation des échanges agricoles selon GTAP-AGR (milliards de \$).

\begin{tabular}{|lccccc|}
\hline Monde & $\begin{array}{c}\text { Pays } \\
\text { à revenus } \\
\text { élevés }\end{array}$ & Total & Bengladesh & Mozambique & $\begin{array}{c}\text { Autre Afrique } \\
\text { subsaharienne }\end{array}$ \\
\cline { 3 - 6 } & 41569 & 11930 & $-0,50$ & $-0,06$ & $-1,67$ \\
\hline 55658 & 4150 & & & & Pays en développement \\
\hline
\end{tabular}

Source : Hertel et Keeney [3]. 
Tableau 3. Répartition des gains de la libéralisation totale des échanges selon Linkage (milliards de \$).

\begin{tabular}{|lccccc|}
\hline Monde & $\begin{array}{c}\text { Pays à revenus } \\
\text { élevés }\end{array}$ & \multicolumn{4}{c|}{ Pays en développement } \\
\cline { 2 - 6 } & Total & $\begin{array}{c}\text { Pays à faibles } \\
\text { revenus }\end{array}$ & $\begin{array}{c}\text { Sélection de pays } \\
\text { d'Afrique } \\
\text { subsaharienne }\end{array}$ & $\begin{array}{c}\text { Autre Afrique } \\
\text { subsaharienne }\end{array}$ \\
\hline 287,3 & 201,6 & 85,7 & 16,2 & 1 & 2,5 \\
\hline
\end{tabular}

Source : Anderson et al. [4].

Tableau 4. Impact de différents scénarios de libéralisation sur la pauvreté (variation du nombre de personnes vivant avec moins de $1 \$$ par jour, en millions).

\begin{tabular}{|lcccc|}
\hline & \multicolumn{1}{|c|}{$\begin{array}{c}\text { Nombre } \\
\text { de pauvres } \\
\text { dans la référence }\end{array}$} & $\begin{array}{c}\text { Libéralisation } \\
\text { totale, } \\
\text { version } \\
\text { dynamique }\end{array}$ & $\begin{array}{c}\text { Libéralisation } \\
\text { totale, } \\
\text { version statique }\end{array}$ & $\begin{array}{c}\text { Scénario } \\
\text { Doha }\end{array}$ \\
\hline $\begin{array}{l}\text { Pays en } \\
\text { développement } \\
\text { (modèle Linkage) }\end{array}$ & 622 & $-31,9$ & $-23,8$ & $-2,5$ \\
$\begin{array}{l}\text { Afrique } \\
\text { Subsaharienne } \\
\text { (modèle Linkage) }\end{array}$ & 340 & $-21,1$ & -16 & $-0,5$ \\
$\begin{array}{l}\text { Cameroun } \\
\text { (modèle GTAP) }\end{array}$ & 6,3 & & $+0,3$ & $-0,02$ \\
$\begin{array}{l}\text { Mozambique } \\
\text { (modèle GTAP) }\end{array}$ & 9 & $+0,06$ & $+0,03$ \\
\hline
\end{tabular}

Source : Pays en développement et Afrique subsaharienne, Anderson et al. [6] p. 518-519 et Anderson et al. [4] p. 382 ; Cameroun et Mozambique : Hertel et Winters [2], p. 27. La pauvreté est définie conformément à la mesure adoptée par les grandes organisations internationales comme caractéristique des individus vivant avec moins de 1 \$ par jour (pauvreté extrême) et moins de 2 \$ par jour (pauvreté). Pour les lignes « Pays en développement » et «Afrique Subsaharienne » l'évaluation concerne l'année 2015 et est réalisée avec le modèle Linkage. Pour le Cameroun et le Mozambique, il s'agit d'évaluations réalisées à partir de modèles d'équilibre général nationaux, selon la méthodologie GTAP.

Tableau 5. Variation de la pauvreté selon différents scénarios de libéralisation (en millions).

\begin{tabular}{|lccc|}
\hline & Doha-standard & Doha-total & Libéralisation totale \\
\hline Malawi & $+0,001$ & $-0,003$ & $-0,045$ \\
Mozambique & $+0,015$ & $+0,013$ & $-0,014$ \\
Ouganda & $+0,016$ & $+0,018$ & $+0,069$ \\
Zambie & $+0,006$ & $+0,004$ & $+0,01$ \\
\hline
\end{tabular}

Source : Ivanic [7].

gains réalisés sur les marchés des pays à hauts revenus sont contrebalancés par des pertes sur les marchés des autres pays en développement et des économies en transition. Exprimées en pourcentage de la valeur ajoutée agricole, ces pertes sont toutefois faibles pour les PMA étudiés : moins de 0,2 \% (Hertel et Keeney, p. 52 et 56 [3]).

Dans le modèle Linkage (tableau 3), de faibles gains apparaissent même pour les pays les plus pauvres suite à la libéralisation totale des échanges. De plus, les scénarios « Doha », où la libéralisation est partielle, indiquent des gains beaucoup plus modestes encore, environ le tiers.

Au-delà des impacts au niveau national, les Objectifs du Millénaire supposent une réduction drastique de la pauvreté et plusieurs éva- pauvreté sont proportionnelles à la croissance économique. C'est donc dans les pays où la croissance générée par les scénarios de libéralisation est importante - Inde et Chine en particulier - que la réduction de la pauvreté est la plus forte. Par ailleurs, les élasticités de la pauvreté sont beaucoup plus faibles, en valeur absolue, pour I'Afrique subsaharienne que pour I'Asie ${ }^{4}$.

Le calcul est différent dans les modèles utilisant la méthodologie GTAP. On procède alors en deux étapes : le modèle mondial GTAP permet tout d'abord d'évaluer les changements dans les prix internationaux engendrés par la libéralisation des échanges; ces variations sont ensuite utilisées comme variables d'entrée dans des modèles nationaux, où l'on calcule la rémunération des facteurs de production et les modifications ainsi engendrées dans les revenus des différents types de ménages. Les conséquences en termes de pauvreté en sont déduites. Les estimations présentées dans le tableau 4 pour le Cameroun et le Mozambique utilisent les techniques de la microsimulation ${ }^{5}$. La diminution de la pauvreté est beaucoup plus faible qu'avec les estimations de Linkage. On a même une croissance du nombre de personnes touchées par ce fléau dans la plupart des cas.

De nombreux autres travaux sur cette question arrivent au même résultat : il ne faut pas attendre de fortes réductions de la pauvreté suite à la libéralisation des échanges ${ }^{6}$.

\section{Pourquoi un impact si faible de la libéralisation sur les pays les plus pauvres?}

Ainsi, d'après les modèles, les impacts de la libéralisation des échanges, au niveau des nations les plus pauvres comme à celui des individus, sont extrêmement faibles.

Cependant, puisque la plupart des pauvres se trouvent en zone rurale et dépendent des activités agricoles pour leur survie, la croissance des prix agricoles devrait à terme leur être favorable, du fait des progrès dans leur rémunération ainsi engendrés, en tout cas pour les ménages vendeurs nets de produits agricoles. Par ailleurs, on attend généralement beaucoup d'un développement du secteur agricole en termes de réduction de la pauvreté. L'agriculture, du fait des gains importants de productivreté). Le nombre de personnes que la libéralisation des échanges permet de «sortir de la pauvreté » est beaucoup plus important selon le modèle Linkage que selon les évaluations réalisées à partir des modèles GTAP. Les résultats en termes de pauvreté restent cependant très modestes pour l'Afrique subsaharienne, et ce particulièrement lorsque la libéralisation est partielle. Ceci s'explique simplement par le mode de calcul utilisé, où les sorties de la

\footnotetext{
${ }^{4}-1,3$ pour l'Afrique subsaharienne et $-3,9$ pour I'Asie du Sud [5].

${ }^{5} C^{\prime}$ est-à-dire que plutôt que de répartir les ménages en plusieurs groupes, selon les niveaux de revenus, on garde l'ensemble de l'échantillon, afin de ne pas perdre d'information.

${ }^{6}$ Une revue bibliographique sur cette question est proposée par Reimer [8].
} 
vité attendus, en particulier dans les pays les plus pauvres, est souvent désignée comme le «moteur » du développement. Elle permet la croissance des emplois et des revenus en milieu rural.

De plus, du fait de la dispersion géographique de la production agricole, l'essor de l'agriculture suppose celui des infrastructures de transport, réduisant ainsi les coûts de transaction, souvent mentionnés comme un obstacle majeur au développement. Enfin, les gains de productivité doivent permettre la diminution des prix des produits alimentaires, élément-clé dans la lutte contre la pauvreté, la population la plus pauvre dépensant généralement plus de $50 \%$ de son budget dans les produits alimentaires. Cet impact sur les prix agricoles permet aussi au développement de l'agriculture d'améliorer la situation des pauvres urbains. Certains auteurs soulignent ainsi les effets multiplicateurs du développement agricole, $1 \$$ de revenu agricole additionnel pouvant engendrer une croissance de $2,5 \$$ dans les pays à bas revenus [9].

Cependant, pour voir apparaitre ce cercle vertueux suite à la libéralisation des échanges, il est nécessaire d'être compétitif au niveau international. Les différences gigantesques de productivité, entre les techniques de production des grands exportateurs, basées sur le recours intensif au capital sur des exploitations de plusieurs centaines d'hectares, et la petite agriculture familiale, qui opère souvent sur moins d'un hectare avec un capital réduit au strict minimum, ne permettent pas à cette dernière d'espérer soutenir la compétition ${ }^{7}$. Dans une telle configuration, non seulement les exportations ne progressent pas mais les importations ont tendance à augmenter suite au retrait des barrières tarifaires. C'est bien ce qui semble se passer dans les modèles dont les résultats ont été présentés ci-dessus : les pays les plus pauvres bénéficient peu de l'accès au marché des pays riches, au contraire des pays émergents (Argentine, Brésil, Chine, Inde).

En principe, un secteur agricole peu compétitif $n^{\prime}$ est pas un obstacle rédhibitoire au développement. Dans les évaluations chiffrées présentées ci-dessus, on suppose que la main$d^{\prime} œ u v r e$ peut être transférée d'un secteur à l'autre, sans aucune difficulté ${ }^{8}$. Ce n'est pourtant pas ce que l'on peut observer pour les pays $\mathrm{d}^{\prime}$ Afrique subsaharienne. II semble que ces derniers ne soient compétitifs dans aucun secteur,

\footnotetext{
${ }_{7}$ Pour les céréales, Mazoyer [10] indique un écart de productivité pouvant aller de 1 à 2000 .

${ }^{8}$ Ceci est justifié par le recours à l'analyse en statistique comparative : on compare deux situations en équilibre, après que tous les ajustements se soient réalisés. Une analyse critique de cette hypothèse sera proposée dans la dernière partie de cet article.
}

si bien qu'ils sont laissés largement à l'écart du développement du commerce et de la redistribution qui en découle.

\section{Des éléments qui font craindre une évolution réelle encore plus défavorable aux PMA}

Mais ce n'est pas tout. Un certain nombre d'éléments non représentés dans les modèles font craindre une évolution beaucoup plus pessimiste encore de la situation des nations les plus défavorisées.

Si I'on attend des gains à la libéralisation des échanges, c'est que l'on suppose que des prix mondiaux, vierges de toute distorsion - taxes et subventions commerciales ou internes assureraient dans chaque nation une allocation plus efficace des ressources productives. C'est ce que les économistes appellent une allocation optimale, conforme aux avantages comparatifs. Trois éléments font craindre que les gains théoriques mentionnés soient difficiles à observer : - l'instabilité des prix sur les marchés mondiaux, qui brouille les signaux censés diriger l'allocation des ressources vers les secteurs les plus efficients dans les différentes nations ;

- l'importance des coûts de transaction dans les pays les plus pauvres, liée en particulier à la faiblesse des infrastructures qui isole les prix sur les marchés locaux des cours internationaux ; - certaines hypothèses réalisées dans la plupart des modèles qui surestiment les gains liés à la libéralisation (le plein-emploi et la perfection de l'information par exemple) et le recours à des techniques d'évaluation qui ignorent les coûts d'ajustement et ainsi les pertes potentielles (statique comparative)

Ces trois points vont être examinés successivement.

Les marchés mondiaux de produits agricoles, dont les variations de prix sont censées permettre une modification des signaux à la source des comportements économiques, sont caractérisés par une forte instabilité : des variations de 20 ou $30 \%$ des prix sont habituelles sur la plupart des marchés. Dans les modèles qui viennent d'être cités, par contre, les hausses estimées des prix atteignent rarement les $10 \%$ (sauf pour certains produits comme le lait et le sucre). II faut alors se demander comment les agents économiques feront la distinction entre cette hausse pérenne des prix et les fluctuations habituelles.

Les auteurs des modèles se reposent sur l'argument selon lequel I'instabilité des prix diminuerait fortement à la suite de la libéralisation des échanges. En effet, lorsque les fluctuations sont générées par des chocs extérieurs au marché, une sécheresse, des attaques de ravageurs, des inondations, l'élargissement des marchés et le retrait des protections des États permettent une réduction significative de l'instabilité-prix. Sur un marché plus large, où les sources d'approvisionnement sont diversifiées, la réduction de la production, résultat du choc dans une région donnée, a toutes les chances d'être compensée par une récolte exceptionnelle dans une autre région. Par ailleurs, les politiques nationales de protection contre l'instabilité des prix internationaux, rejettent leurs déséquilibres internes sur les marchés extérieurs, en ajustant leurs importations et exportations au niveau des récoltes domestiques, ce qui a pour effet d'accroître l'instabilité des prix sur les marchés mondiaux?

Tout cela est indiscutable pour ce qui est des fluctuations générées par des chocs extérieurs, comme les accidents climatiques. Toutefois, de nombreux travaux sur la nature de l'instabilité des prix tendent à montrer qu'à côté de ce phénomène il en existe un autre, lui aussi générateur de fluctuations. II tient aux imperfections de l'information, conjuguées avec la rigidité de la demande. Or, ces deux facteurs jouent un rôle majeur dans la détermination des prix ${ }^{10}$. Contrairement à celles qui sont produites par des chocs exogènes, ces fluctuations dites endogènes $n^{\prime}$ ont aucune chance de disparaître avec l'élargissement du marché. Dans la

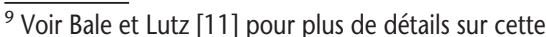
question. Tyers et Anderson, p. 61 [12] évaluent les gains à en attendre de la manière suivante: « (...) les fluctuations des prix internationaux des produits alimentaires sont imputables à concurrence de $25 \%$ environ aux aspects stabilisateurs de la politique agricole commune de la CEE (...) Autrement dit, si les pays de l'OCDE et les pays en voie de développement libéralisaient intégralement leurs marchés, les fluctuations des marchés internationaux seraient réduites à un tiers de ce qu'elles sont actuellement. (...)». ${ }^{10}$ Voir par exemple Mandelbrot ([13] p. 330). Celui-ci souligne la fréquence du « hasard sauvage » dans les séries économiques et en particulier dans les séries de prix, « (...) la raison, à priori est que l'offre et la demande, qui en principe doivent déterminer un prix, résultent toutes deux à la fois de facteurs objectifs et d'anticipations. Même si on accepte pour les premiers une approximation continue, les seconds peuvent changer du tout au tout à la suite d'un signal physique dont la durée et l'énergie sont négligeables, tel le proverbial «trait de plume ». Du coup la rationalité dans la détermination des prix risque fort de mener à des sauts, signifiant qu'elle est déstabilisante ». Ce rôle central des anticipations se trouve déjà chez Keynes [14] qui affirme leur caractère incertain, subjectif et fluctuant. II est à l'origine des politiques agricoles modernes, initiées par Franklin D. Roosevelt au milieu des années 30, et qui étaient justifiées par de telles considérations [16]. On le retrouve enfin dans l'analyse des épisodes fébriles sur
} les marchés financiers. 
meilleure des hypothèses, celui-ci ne fait que les synchroniser (Boussard et al. 2005). Si les fluctuations de prix actuelles sont bien le résultat de ces deux phénomènes, les marchés mondiaux resteront instables. Étant donné la faiblesse des croissances de prix estimés par les modèles mondiaux, il est probable que les impacts de la libéralisation seront difficiles à distinguer des fluctuations habituelles.

Les coûts de transaction recouvrent l'ensemble des coûts d'intermédiation du producteur au consommateur, comme les coûts de transports mais aussi la rémunération des risques endossés par les commerçants. Ceux-ci sont particulièrement élevés dans les pays les plus pauvres du fait de la faiblesse des infrastructures : routes, entrepôts de stockages, marchés organisés. Les coûts de transport sont ainsi en moyenne plus de trois fois plus élevés en Afrique qu'en Asie, et on voit couramment des endroits où le prix double entre la frontière et le marché de destination.

Du fait de ces coûts, les marchés sont peu connectés entre eux et sont soumis à de fortes fluctuations. La moindre variation des quantités offertes a en effet beaucoup d'impact sur les prix à cause de la rigidité de la demande, et de la faiblesse des volumes offerts. Dans une telle situation, l'activité d'intermédiation est risquée, le prix peut en effet «se retourner» pendant le transport des marchandises d'un marché à l'autre, ne permettant plus à l'intermédiaire ou à «l'arbitragiste » de couvrir ses coûts.

Dans ces conditions, on sait que les agents économiques prévoient une prime de risque, ce qui renchérit les marges et ainsi l'écart entre les prix au producteur et au consommateur. Cela diminue les incitations à l'échange et pousse les volumes échangés à la baisse. On se trouve alors dans un cercle vicieux où la faible densité de population associée à la faiblesse des volumes transitant par le marché augmentent les prix aux consommateurs et réduisent les prix offerts aux producteurs, diminuant ainsi les incitations à échanger sur le marché, ce qui réduit le volume des transactions et augmente encore les coûts de transactions ${ }^{11}$. Dans de telles circonstances, les acteurs économiques ont tendance à se protéger des fluctuations en minimisant leurs rapports avec le marché, on reste ainsi dans une économie de subsistance. La "trappe à pauvreté », caractéristique de nombreux pays, parmi les plus pauvres ${ }^{12}$, s'explique ainsi. Ces pays ont généralement une faible productivité du travail dans I'agriculture, où l'essentiel de la main-d'œuvre est cependant employé. La faiblesse de la produc-

\footnotetext{
${ }_{11}$ Voir par exemple Dorward et al. [15].

${ }^{12}$ Voir par exemple Sachs et al. [16].
}

tivité peut s'expliquer par l'absence de capital, qui trouve sa source dans l'absence d'une épargne suffisante liée à la faiblesse des revenus... qui provient de la faiblesse de la productivité. Les coûts de transaction et la faiblesse des infrastructures et plus généralement des « biens publics » renforcent le phénomène, en réduisant la part du prix qui va au producteur $d^{\prime} u n e$ part, et en créant un environnement peu favorable à l'investissement d'autre part.

Ces coûts de transaction définissent par ailleurs une bande de prix à l'intérieur de laquelle il n'y a pas de réactions des ménages vivant de l'agriculture aux variations de prix [17]. II y a alors peu de chance que la croissance des prix agricoles estimée par les modèles mondiaux ait un impact quelconque sur les agriculteurs pauvres des pays à faible revenus.

Plusieurs autres hypothèses des modèles d'équilibre général font aussi craindre une surestimation des gains dans les pays les moins avancés. L'hypothèse de plein-emploi de la main-d'œuvre permet son transfert de secteurs où elle est peu efficace vers des secteurs où sa productivité est plus élevée. Ni les difficultés liées à la qualification, ni la possibilité de passage d'un secteur d'activité au chômage ne sont prises en compte ${ }^{13}$.

De même, la représentation du fonctionnement des marchés agricoles pose problème. Alors que l'instabilité des prix est reconnue comme une caractéristique majeure de ces marchés, elle est ignorée dans les modèles. Ses conséquences sur la production agricole sont pourtant largement documentées ${ }^{14}$ : elle se traduit par une production et un investissement plus faibles, du fait du risque associé à l'activité et de l'instabilité de la rémunération des facteurs ainsi engendrée. Ainsi, l'instabilité des prix est doublement dommageable à la lutte contre la pauvreté : non seulement, la production étant plus faible, les prix sont plus élevés, ce qui est particulièrement négatif pour les consommateurs les plus pauvres; mais en plus, en s'opposant à l'investissement agricole, elle empêche les progrès dans la productivité du travail qui serait permis par la croissance du capital. Le modèle ID3, qui inclut les imperfections du fonctionnement des marchés agricoles, montre que dans ces conditions des pertes de bien-être apparaissent, du fait de l'instabilité des prix et des erreurs dans les prévisions des producteurs qui en découlent (encadré 1).

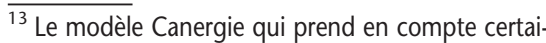
nes imperfections du marché du travail trouve des gains beaucoup moins importants.

${ }^{14}$ Pour plus de détails, voir par exemple Boussard [18].
}

Par ailleurs, la plupart des modèles utilisés pour ces estimations utilisent la statique comparative, c'est-à-dire que l'on compare deux situations d'équilibre, en l'occurrence "avec » et « sans » libéralisation, à un horizon de temps non spécifié précisément mais où tous les ajustements ont déjà pris place. On ignore alors par définition les coûts de ces ajustements. Or, dans de multiples cas, les changements institutionnels et économiques se traduisent par des difficultés d'adaptation, des structures comme des agents, qui peuvent être extrêmement longues et coûteuses sur le plan humain [19]. II est donc important que les modèles soient dynamiques, afin de permettre d'évaluer les trajectoires des économies d'un équilibre à l'autre et leurs conséquences sur le bien-être des populations. Les effets du risque et de la contrainte de liquidité ne sont pas seulement à court terme. Au contraire, en l'absence de crédit, l'investissement est souvent le résultat de plusieurs années d'épargne, tandis qu'une « mauvaise » année se traduira par une contrainte de trésorerie sévère, voire des « ventes de détresses » d'une partie des actifs, qui aura des conséquences durables sur l'exploitation.

Enfin, il faut dire un mot des difficultés associées à la validation de ces modèles. Celles-ci tiennent, $d$ 'une part, à leur nature statique et, d'autre part, au fait que par définition les chocs exogènes - telles les incidents climatiques, une variation de la conjoncture extérieure dans un modèle national, le progrès technique - ne sont pas considérés dans les modèles. Après coup, on aura ainsi tendance à expliquer les écarts entre le modèle et la réalité par ces perturbations extérieures à la représentation. Ils deviennent alors très difficiles à valider ou infirmer. De ce fait, relativement peu de travaux satisfaisants ont été réalisés dans ce domaine. Cela semble pourtant de la première importance étant donné la forte variabilité des estimations et l'importance que ces outils semblent avoir dans les négociations.

Finalement, alors que dans la réalité des pays à bas revenus de multiples imperfections de marché - absence de crédit, d'infrastructure, de marché d'assurance, instabilité des prix - se sont révélées comme autant d'obstacles à la croissance économique [21], ces questions sont totalement ignorées dans la plupart des modèles. Ceci s'explique par les simplifications nécessaires et la difficulté à envisager dans des outils réalisés à l'échelle mondiale, la variété des conditions propre à chaque pays. Dans les modèles nationaux, on se heurte à la disponibilité et à la qualité des données, en particulier dans les pays à faibles revenus où les instituts statistiques sont souvent peu nombreux. 
Malgré cela, de plus en plus d'efforts sont réalisés pour représenter les imperfections des marchés. Ces efforts permettent d'affiner les estimations de bénéfices de la libéralisation, et amènent à les revoir à la baisse $e^{15}$.

En définitive, si les estimations présentées ici, tant au niveau des gains nationaux pour les pays à faibles revenus que sur le plan de la réduction de la pauvreté, sont peu optimistes, les caractéristiques des modèles utilisés font craindre une surestimation des gains. II est donc probable que les conséquences de la libéralisation des échanges soient encore moins favorables aux PMA.

\section{Conclusion}

Un scénario pessimiste se dessine ainsi pour les pays les plus pauvres. On peut craindre que les tendances actuelles se maintiennent, c'est-àdire que la libéralisation des échanges ne se traduise par aucune amélioration de la situation pour les populations pauvres des pays à faibles revenus, mais plutôt par une croissance des importations pour nourrir les urbains tandis que les agricultures se replieront sur une autosubsistance qui ne leur permettra pas de sortir de la « trappe à pauvreté ». Cette tendance à la dégradation de la situation des pays à faible revenu, en particulier en Afrique, tandis que les progrès en matière de pauvreté sont concentrés dans certains pays émergents (Inde et Chine) est caractéristique de la situation actuelle [25]. Se pose alors la question de la redistribution internationale qui pourrait prendre place pour permettre de lutter contre cette situation. En réalité, quatre modes de redistribution internationale sont à l'œuvre à l'heure actuelle: I'aide publique au développement, les investissements directs à l'étranger, le commerce et les migrations. II semble que l'aide publique au développement, du moins dans sa forme et son niveau actuels, ne permette pas de relever les défis de la lutte contre la pauvreté dans les pays à faibles revenus. Les investissements directs à l'étranger y sont généralement plus faibles qu'ailleurs, du fait d'un environnement peu favorable aux activités économiques - absence d'infrastructures de transport, faiblesse des réseaux électriques et de communication, fonctionnement peu satisfaisant des marchés, absence de demande solvable. La libéralisation du commerce ne semble pas devoir profiter aux pays les moins avancés. Reste alors les migrations. Et c'est bien ce qu'on observe dans plusieurs pays parmi les plus pau-

\footnotetext{
${ }^{15}$ Voir par exemple Arndt [20].
}

vres où les seuls ménages qui voient leur situation économique et nutritionnelle s'améliorer sont ceux où certains membres de la famille ont migré et effectuent des transferts.

\section{RÉFÉRENCES}

1. WTO. Doha Ministerial Declaration. WT/MIN(01)/DEC/1, 2001.

2. HERTEL TW, WINTER LA. Poverty Impact of a WTO agreement : Synthesis and Overview. In : Hertel W, ed. Poverty and the WTO : Impacts of the Doha development Agenda. Washington: World Bank, 2006.

3. HERTEL TW, KEENEY R. What is at stake : the relative importance of imports barrier, export subsidies and domestic support. In : Anderson, Mardin, eds. Agriculture, Trade Reform and the Doha development Agenda. New York: Macmillan, 2006.

4. ANDERSON K, MARTIN W, VAN DER MENSBRUGGHE D. Market and Welfare Implications of Doha Reform Scenarios. In : Anderson, Mardin, eds. Agriculture, Trade Reform and the Doha development Agenda. New York: Macmillan, 2006.

5. CHEN S, RAVAILLON M. How have the world poorest fared since the early 1980's, World Bank Policy Research Working Paper 3341, 2004.

6. ANDERSON K, MARTIN W, VAN DER MENSBRUGGHE D. Global Impacts of the Doha Scenarios on Poverty. In: Hertel, Winters, eds. Poverty and the WTO : Impacts of the Doha development Agenda. Washington: World Bank, 2006.

7. IVANIC M. The effect of a prospective multilateral trade reform on poverty in developing countries. In : Hertel, Winters, eds. Poverty and the WTO: Impacts of the Doha development Agenda. Washington: World Bank, 2006.

8. REIMER JJ. Estimating the poverty impacts of trade liberalization. GTAP working Paper $n^{\circ} 20$. Purdue University, 2002.

9. PINSRUP-ANDERSEN P, LUNDBERG M, GARETT IL. Foreign Assistance to Agriculture : A win-win Proposition. Washington DC : IFPRI, 1995.

10. MAZOYER M. Protéger la paysannerie pauvre dans un contexte de mondialisation. Rome: FAO, 2001.

11. BALE M, LUTZ E. The effect of Trade intervention on international price instability. AJAE $1979 ; 61$.

12. TYERS K, ANDERSON JK. Disarray in Worl Food Markets: a quantitative assessment. Cambridge : Cambridge University Press, 1992.
13. MANDELBROT $B$. Le syndrome de la variance infinie et ses rapports avec la discontinuité des prix. Economie Appliquée 1973 ; 26(1) : 321-48.

14. KEYNES JM. A treatise of probability. Macmillan, 1921.

15. DORWARD A, KYDD J, MORRISON J, UREY I. A policy Agenda for pro poor Agricultural Growth. World development 2004; 32(1): 73-89.

16. SACHS JD, MCARTHUR JW, SCMIDT-TRAUB G, ET AL. Ending Africa Poverty Trap. Brooking papers on economic activity 2004; 1 . Washington DC: Brookings Institution Press. http ://www. unmilleniumproject. org/ reports/secretariatdocs. htm.

17. KEY N, SADOULET E, DE JANVRY A. Transactions costs and agricultural household supply response. Am J Agricultural Econom, 82 : 254259.

18. BOUSSARD JM. Economie de l'Agriculture. Paris : Economica, 1987.

19. STIGLITZ JE, CHARLTON A. Un cycle de négociations commerciales pour le développement? Rev Econ Dev 2005 ; 4 : 18-54.

20. ARNDT C. The Doha Round and the Mozambique. In : Hertel, Winters, eds. Poverty and the WTO: Impacts of the Doha development Agenda. Washington: World Bank, 2006.

21. KHERALLAH M, DELGADO C, GABREMATHIEU E, MINOT N, JOHNSON M. The Road Half Travelled: Agricultural Market Reform in Subsaharian Africa. Washington DC : IFPRI, Food policy report, 2000.

22. BOUSSARD JM, GERARD F, PIKETTY MGP. Libéraliser l'agriculture mondiale? Théories modèles et réalités. CIRAD, 2005.

23. BOUSSARD JM, GERARD F, PIKETTY MG, VOITURIEZ T, CHRISTENSEN A. May the pro- poor impacts of trade liberalization vanish because of imperfect information? Agric Econ 2004 ; 31(2) : 297-305.

24. AYOUZ M, BOUSSARD JM, GERARD F, PIKETTY MG, VOITURIEZ T. Libéralisation, agriculture et pauvreté : se peut-il que les bénéfices de la libéralisation soient absorbés par l'imperfection des marchés? OCL 2004 ; 11(4-5) : 345534.

25. BOURGUIGNON. Distribution et redistribution mondiales : une vue préliminaire. Rev Econ Dev $2005 ; 4: 6-15$.

\section{Pour en savoir plus}

ACKERMAN F. The Shrinking Gains from Trade. Medford, MA : Tufts University, 2005 ; (Global Development and Environment Institute, WP 05-01)

POLANSKI S. Winners and losers : impact of the Doha round on developing countries. Canergie Endowment for International Peace, 2006. 